\title{
A METHOD FOR DETERMINING THE CHEMICAL COMPOSITION PARAMETERS $(X, Y, Z)$ OF GALACTIC CLUSTERS
}

\author{
WAYNE OSBORN and JUAN J. CLARIÁ* \\ Instituto Venezolano de Astronomia, Mérida, Venezuela
}

\begin{abstract}
A method is described by means of which the chemical composition parameters $(X, Y, Z)$ may be derived for galactic clusters that contain evolved red giant members and for which the cluster reddening and distance moduli are known. The method is based on observations of the evolved stars utilizing the DDO intermediate-band system. First, the DDO photometry by itself allow's one to separate with a high degree of certainty the physical members of the clusters from the field stars. In cases where membership in the cluster can be confirmed, the unreddened DDO colours can be used to obtain the effective temperatures, surface gravities and metal abundances $(Z)$ of the stars. These data combined with the absolute magnitudes available from the distance modulus permit the masses to be calculated. Finally, if some of the observed red giants form part of a clump on the giant brach in the HR diagram, the helium abundance $(Y)$ of the clusters can be estimated by comparing the derived physical parameters of these stars with theoretical models. An example of the application of the method to the galactic cluster NGC 2420 is given.
\end{abstract}

\section{Introduction}

In a recent study Gross (1973) showed that the position of a zero age horizontal branch (ZAHB) star in the effective temperature-surface gravity plane is determined almost entirely by its helium abundance. This provides a simple method for estimating the helium abundances of several types of objects, for example the globular clusters. Applying this theoretical result to the small amount of observational data available Gross found the unexpected result that population I objects appear to have smaller helium abundances than population II stars. A compilation of all published helium abundance determinations seemed to support this conclusion, but it was obvious that more and better data would be needed for a definitive answer.

One obvious way to test Gross' results is to compare the results from applying his helium abundance method to globular cluster horizontal branch stars and to their population I analogues the clump stars of evolved galactic clusters. A number of determinations of temperatures and gravities for globular cluster horizontal branch stars have been published (Newell et al., 1969a, 1969b; Philip, 1972; Osborn, 1973) but little data is available for clump stars. It was therefore felt it would be worthwhile to attempt to derive temperatures and gravities for clump stars in evolved galactic clusters. Rather than present definitive results, the object of this paper is to show what has been learned from

\footnotetext{
* Visiting astronomer of Cerro Tololo Inter-American Observatory, operated by the Association of Universities for Research in Astronomy, Inc., under contract with the National Science Foundation.
} 
some exploratory steps in this direction using the DDO intermediate-band system (McClure and van den Bergh, 1968; McClure, 1973). In particular, we will describe a procedure that can be used to determine the chemical composition parameters of clusters with clump stars for which the reddening and distance modulus are known.

\section{Identification of the Clump Stars}

The first problem that one encounters is that of separating red field stars from the physical members of the clusters. The best method for doing this is probably on the basis of proper motions and/or radial velocities, but frequently these data are unreliable or not available. We have found that membership can usually be assigned with fair certainty using only $U B V$ and DDO photometry. The DDO $C(45-48)-C(42-45)$ and $C(41-42)$ $-C(42-45)$ diagrams usually allow a decision to be made on the basis of the photometric spectral type and the distance modulus obtained from the corresponding $M_{v}$ (Janes, 1975). For example, foreground dwarfs usually are easily identified as shown in Figure 1. In regions of heavy interstellar reddening the situation can become more complicated. In such cases, however, the reddening itself can be used as a criterion. Assuming the Whitford reddening curve, McClure and Racine (1969) have derived the relation valid for giants with $0.80 \leqslant(B-V)_{0} \leqslant 1.55$ :

$$
E(B-V)=2.175(B-V)-2.380 C(45-48)-1.420 C(42-45)+1.841
$$

where all the photometric indices are the observed (reddened) values. Figure 2 shows a comparison for several clusters of reddenings derived using this equation with those from main sequence fitting from the literature. Thus, the probability of membership can be estimated from how closely the computed reddening of a given star agrees with the value accepted for the cluster. Examples of how this procedure can be applied are shown in Table I for two clusters. The columns of the table give the accepted reddening and distance modulus of the cluster, the observed magnitude of the star and the predicted spectral type if the star is a cluster member, and the parameters obtained from our DDO photometry. The values in parenthesis are those that result if the cluster reddening is used rather than the DDO determination. For NGC 2269 it is clear that star 1 is a foreground star and star 4 a probable cluster member. For NGC 2972 star 11 is probably a member, stars 2 and 14 background supergiants.

The next step is to identify which of the cluster members are the clump stars. No satisfactory solution to this problem has yet been found and so far the procedure followed has been to observe and reduce all stars in the neighbourhood of the clump and then make the selection on the basis of the agreement in the derived temperatures, gravities, and bolometric magnitudes. 


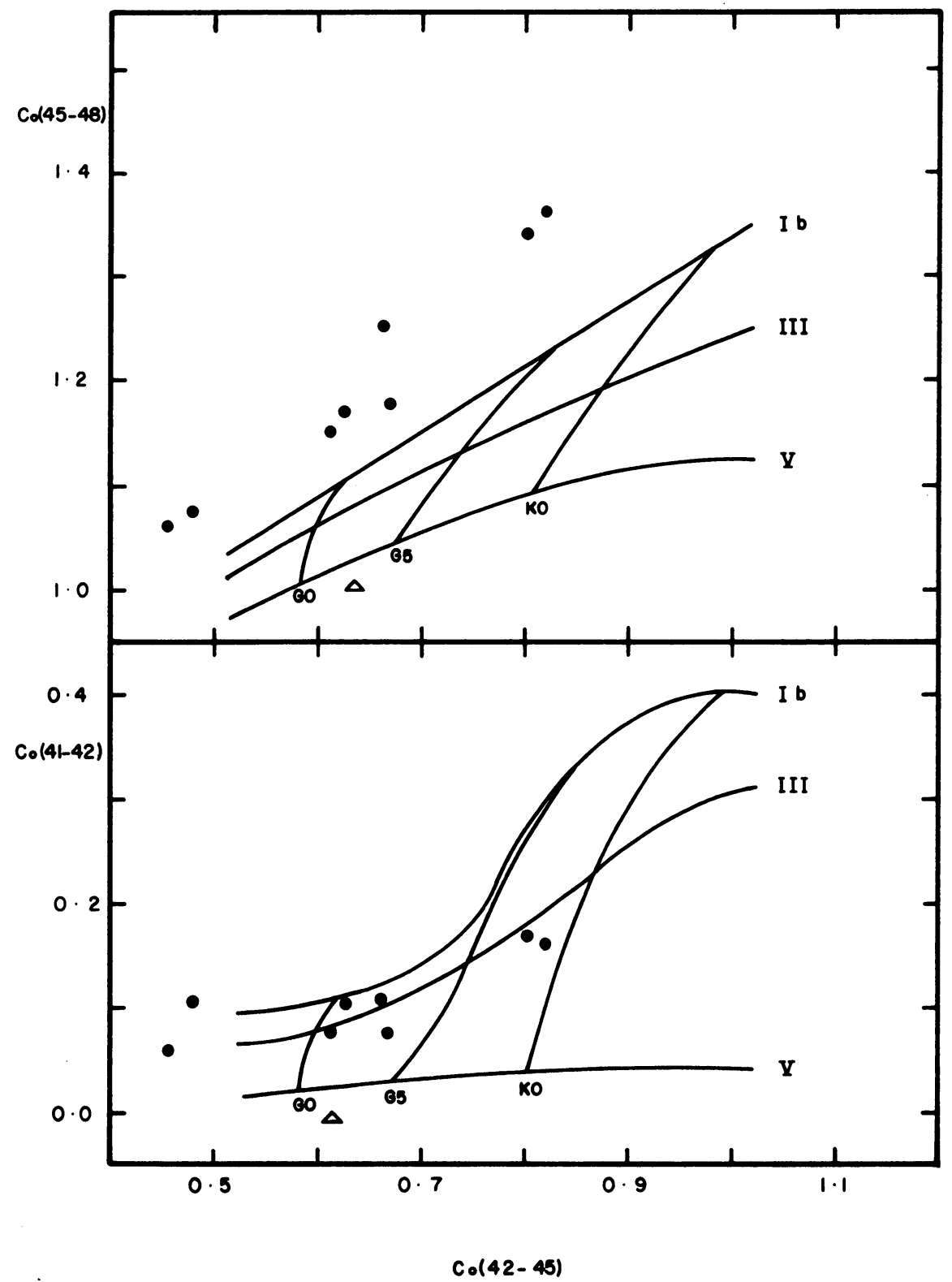

Fig. 1. Observed colours of several stars in M92. One of the stars (triangle) is shown by the DDO colours to be a foreground $\mathbf{G}$ dwarf.

\section{The Effective Temperature and Surface Gravities}

Let us now assume that we can say with some certainty what the interstellar reddening of 


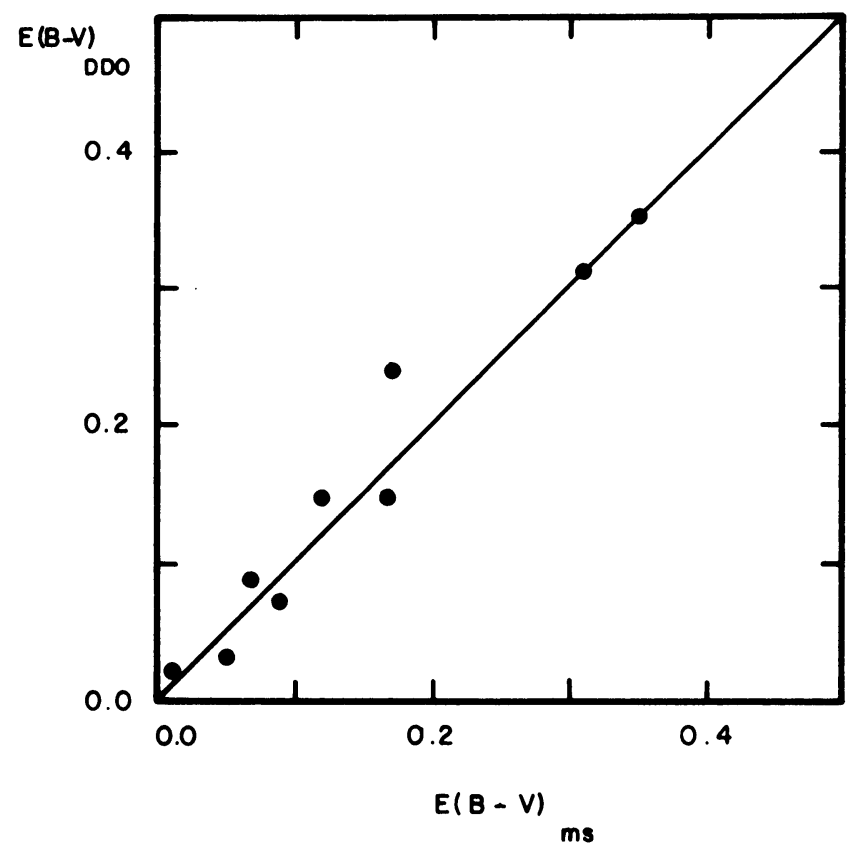

Fig. 2. A comparison between the reddening values found from DDO photometry with those from main sequence fitting for several open clusters.

TABLE I

\begin{tabular}{|c|c|c|c|c|c|c|c|c|c|}
\hline \multirow[b]{2}{*}{ NGC } & \multirow[b]{2}{*}{$E(B-V)$} & \multirow[b]{2}{*}{$V-M_{v}$} & \multirow[b]{2}{*}{ Star } & \multirow[b]{2}{*}{$V$} & \multirow[b]{2}{*}{$\begin{array}{l}\text { Predicted } \\
\text { sp. type }\end{array}$} & \multicolumn{4}{|c|}{ Results from DDO photometry } \\
\hline & & & & & & $E(B-V)$ & $V-M_{\nu}$ & & DDO Spectral type \\
\hline \multirow{2}{*}{2269} & 0.19 & 10.7 & 1 & 8.6 & G9 II & 0.01 & 8.6 & (7.2) & K1 III (K pec) \\
\hline & & & 4 & 8.2 & G8 II & 0.18 & 10.7 & $(10.7)$ & G5 Ib-II (G5 Ib-II) \\
\hline \multirow[t]{3}{*}{2972} & 0.35 & 11.5 & 2 & 11.4 & K1 II-III & 0.30 & 17.4 & $(16.8)$ & G8 Ib (G8 Ib) \\
\hline & & & 11 & 12.0 & G8 II-III & 0.29 & 11.7 & (11.1) & G8 III (G8 III) \\
\hline & & & 14 & 9.4 & K3 II & 0.45 & 15.1 & (15.7) & K0 Ib (K3 Iab) \\
\hline
\end{tabular}

the cluster is and which are the physical members. We correct the observed DDO colours for reddening. The DDO $C(45-48)-C(42-45)$ two colour diagram has been empirically calibrated in terms of effective temperature and surface gravity (Figure 3 ) and therefore the unreddened indices give directly the desired physical parameters. The calibration, however, is only valid for stars of normal, i.e. approximately solar metal abundance. For stars with significantly non-solar abundance the colour indices must be corrected to account for the different degree of line blanketing:

$$
\text { COLOUR }_{\text {normal }}=\text { COLOUR }_{\text {observed }}-A[\mathrm{Fe} / \mathrm{H}]
$$




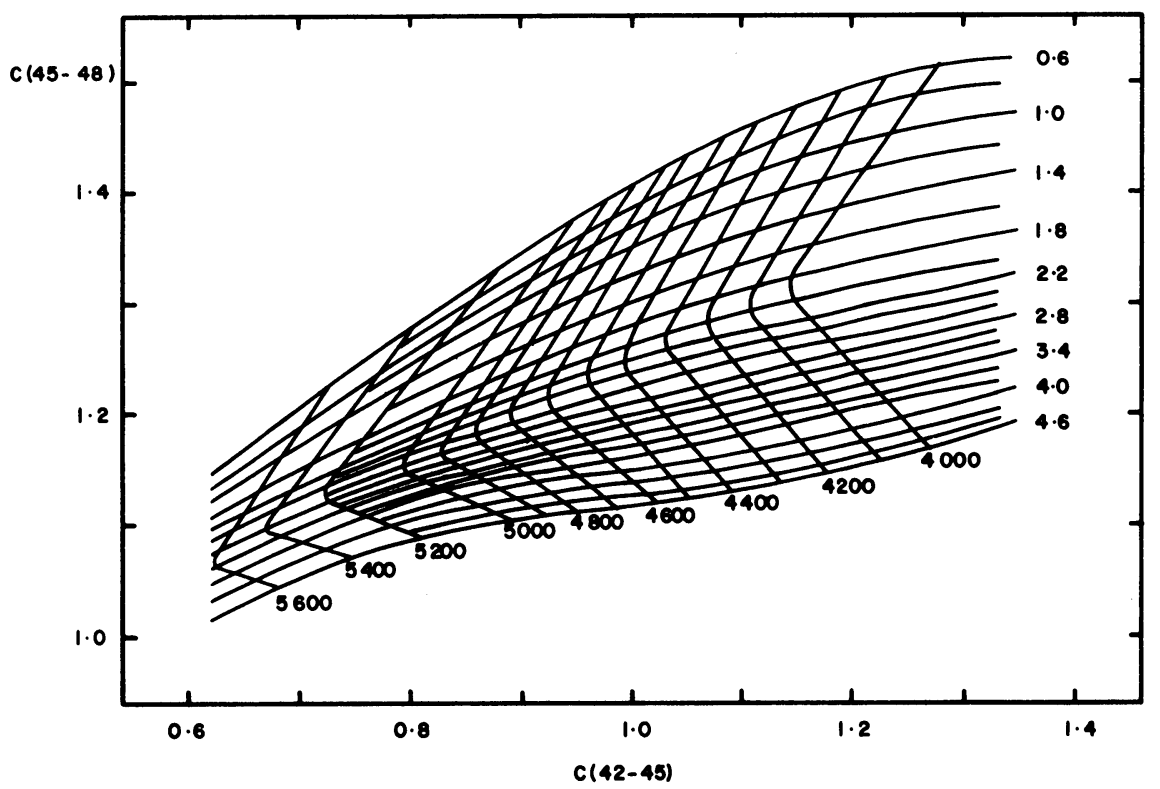

Fig. 3. The relationship between the DDO colour indices and effective temperature $\left(T_{\text {eff }}\right)$ and surface gravity $(\log g)$.

The correction factors $A$ have been empirically determined and tabulated by Osborn (1973). To determine $[\mathrm{Fe} / \mathrm{H}]$ use is made of the DDO $C(41-42)$ index. Janes (1975) has tabulated the average value of this index as a function of $C(45-48)$ and $C(42-45)$ and has shown that the cyanogen anomaly $\delta \mathrm{CN}$, defined as the difference between the observed $C(41-42)$ of a star and the normal value for the observed $C(45-48)$ and $C(42-45)$, is well correlated with $[\mathrm{Fe} / \mathrm{H}]$ :

$$
[\mathrm{Fe} / \mathrm{H}]_{\mathrm{DDO}}=4.5 \delta \mathrm{CN}-0.2
$$

This equation is used to derive $[\mathrm{Fe} / \mathrm{H}]$ and the resulting value used to correct the observed $C(45-48)$ and $C(42-45)$ indices so that the temperature-gravity calibration can be applied. The derived $[\mathrm{Fe} / \mathrm{H}]$ can also be adopted as an indication of the heavy element abundance $Z$.

\section{The Helium Abundance}

The effective temperature and surface gravity of the clump stars can now be used to obtain the helium abundances using Gross' theoretical results (Figure 4). It is seen in the figure that provided the mass of the star is less than about $1.0 M_{\odot}$ the determination is insensitive to the core mass, the metal abundance, or the total mass. Evolution of the star is also parallel to the ZAHB lines. One can check if the mass of the observed star is less 


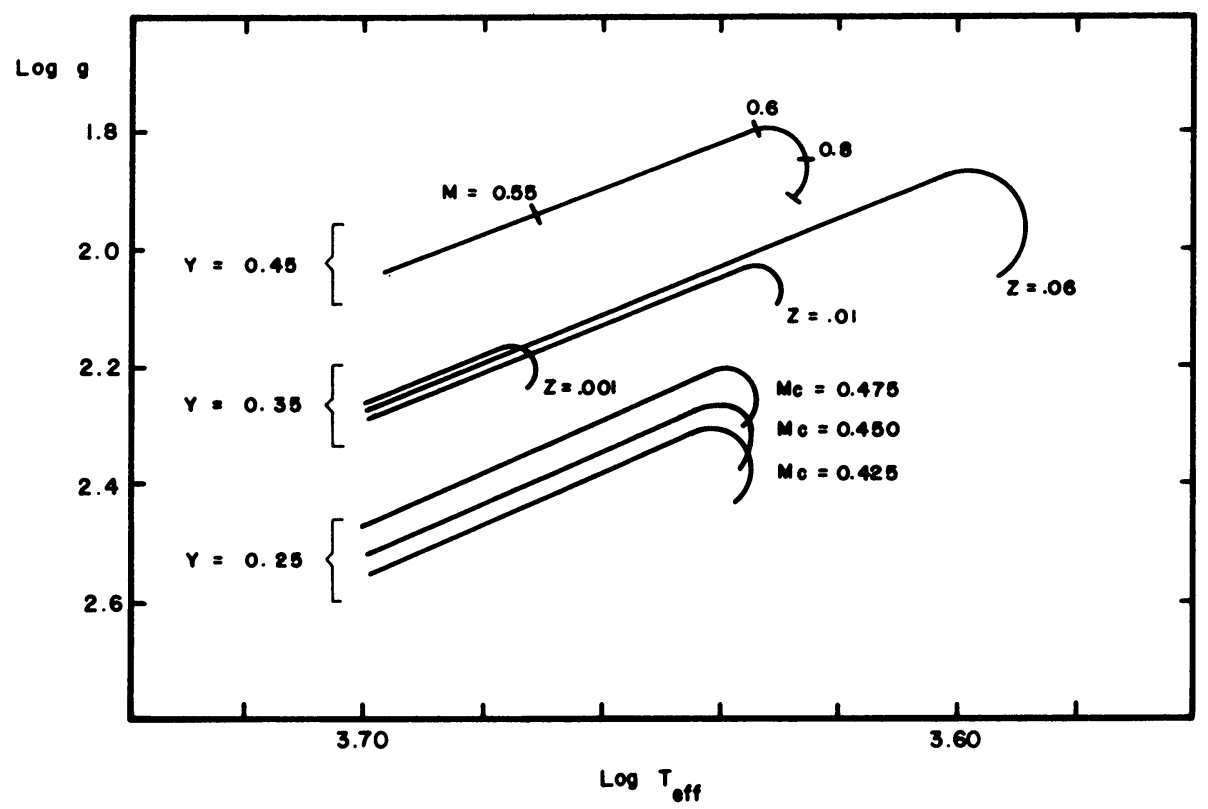

Fig. 4. The position of the zero age horizontal branch in the effective temperature-surface gravity plane based on Gross' models. The dependences on the helium abundance and on the total mass (upper), metal abundance (middle), and core mass (lower) are indicated. It is seen that the helium abundance is the dominant parameter.

than $1.0 M_{\odot}$ if the distance modulus, hence the absolute magnitude of the star, is known. Combining the Stephan-Boltzmann equation and the definition of surface gravity an expression for the mass in terms of the effective temperature, surface gravity and absolute magnitude can be derived (Osborn, 1975).

$$
\log M / M_{\odot}=\log g-4 \log T_{\text {eff }}-0.4 M_{\text {bol }}+12.49
$$

If the mass is in the permitted range then the helium abundance is determined by the adopted temperature and gravity.

A much stronger case can be made by requiring that the results be internally consistent. For example, not only must the derived mass be less than $1.0 M_{\odot}$ but it also must be greater than about $0.4 M_{\odot}$ or it is impossible to form a horizontal branch star. This consideration implies that once the surface gravity and effective temperature have been specified the bolometric magnitude and therefore the distance modulus of the cluster are fixed within rather narrow limits. If the data are consistent this distance modulus will (1) lead to a position of the star in the $M_{\mathrm{bol}}-T_{\text {eff }}$ plane, which is also a function of $Y$ as well as of $Z$, consistent with the previously adopted values and (2) also place the cluster main sequence in a reasonable position in the theoretical HR diagram.

The use of these restrictions to obtain the most consistent estimate of the cluster abundance can be illustrated by an example from a study of NGC 2420. DDO photometry has been published for 11 stars of the cluster of which five form part of the clump on the 
giant branch. The derived data for the five clump stars are listed in Table II. The accepted reddening, true distance modulus, and metal abundance of the cluster are (McClure et al., 1974): $E(B-V)=0.02, V_{0}-M_{v}=11.4$ and $[\mathrm{Fe} / \mathrm{H}]=-0.4(Z \approx 0.01)$. The mean values for the five clump stars are

$$
\log T_{\text {eff }}=3.67 \quad \log g=2.4 \quad M_{\text {bol }}=+0.7 \quad M=0.8 M_{\odot}
$$

TABLE II

\begin{tabular}{lllll}
\hline Star & $\log T_{\text {eff }}$ & $\log g$ & $M_{\text {bol }}$ & $M / M_{\odot}$ \\
\hline 1 & 3.671 & 2.0 & +0.51 & 0.4 \\
2 & 3.670 & 2.6 & +0.69 & 1.3 \\
3 & 3.670 & 2.7 & +0.72 & 1.7 \\
4 & 3.655 & 2.2 & +0.73 & 0.6 \\
5 & 3.672 & 2.4 & +0.79 & 0.8 \\
\hline
\end{tabular}

From the average temperature and gravity and Figure 4 we find a helium abundance of $Y \approx 0.25$. However, adopting $Z=0.01$ we find from the position in the $M_{\mathrm{bol}}-T_{\text {eff }}$ diagram (Figure 5) that $Y \approx 0.35$, in disagreement with the former value.

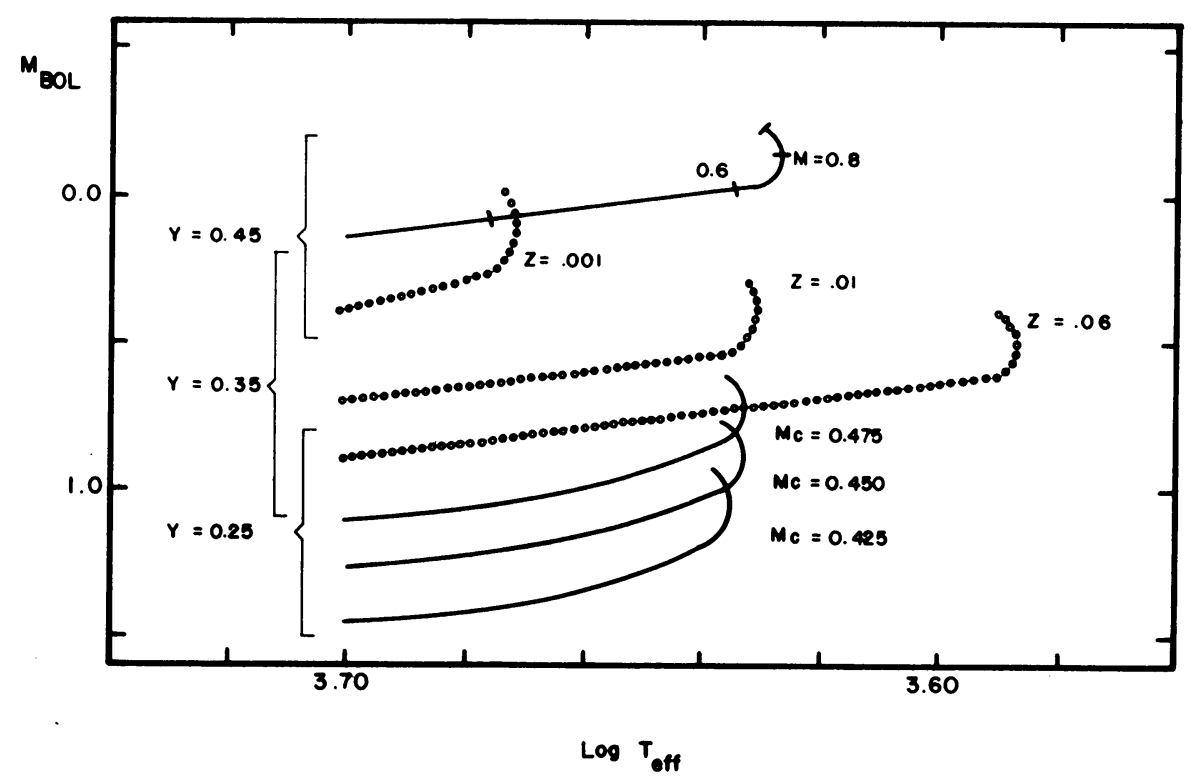

Fig. 5. The position of the zero age horizontal branch in the $M_{\text {bol }}$-effective temperature plane. As in the previous figure, the dependences on helium abundance and on total mass, metal abundance, and core mass are shown. In this diagram the effect of a change in helium abundance can be confused by changes in the other parameters.

Agreement between the two could be obtained by assuming a distance modulus about 0.4 smaller but this change would imply a helium abundance for the main sequence stars 
of $Y \approx 0.45$ (see Figure 6 ) and some of the derived masses become uncomfortably small. The same difficulties are encountered if one tries altering the adopted $Z$. A consistent case can be made by assuming that the two stars with high masses are red giants. Then the average values of the true clump, i.e. horizontal branch, stars are

$$
\log T_{\text {eff }}=3.67 \quad \log g=2.2 \quad M_{\text {bol }}=+0.7 \quad M=0.6 M_{\odot}
$$

These values lead to $Y=0.34$ (Figure 4) and $Y=0.33$ (Figure 5) and the main sequence position is consistent with $Y=0.35$ and $Z=0.01$.

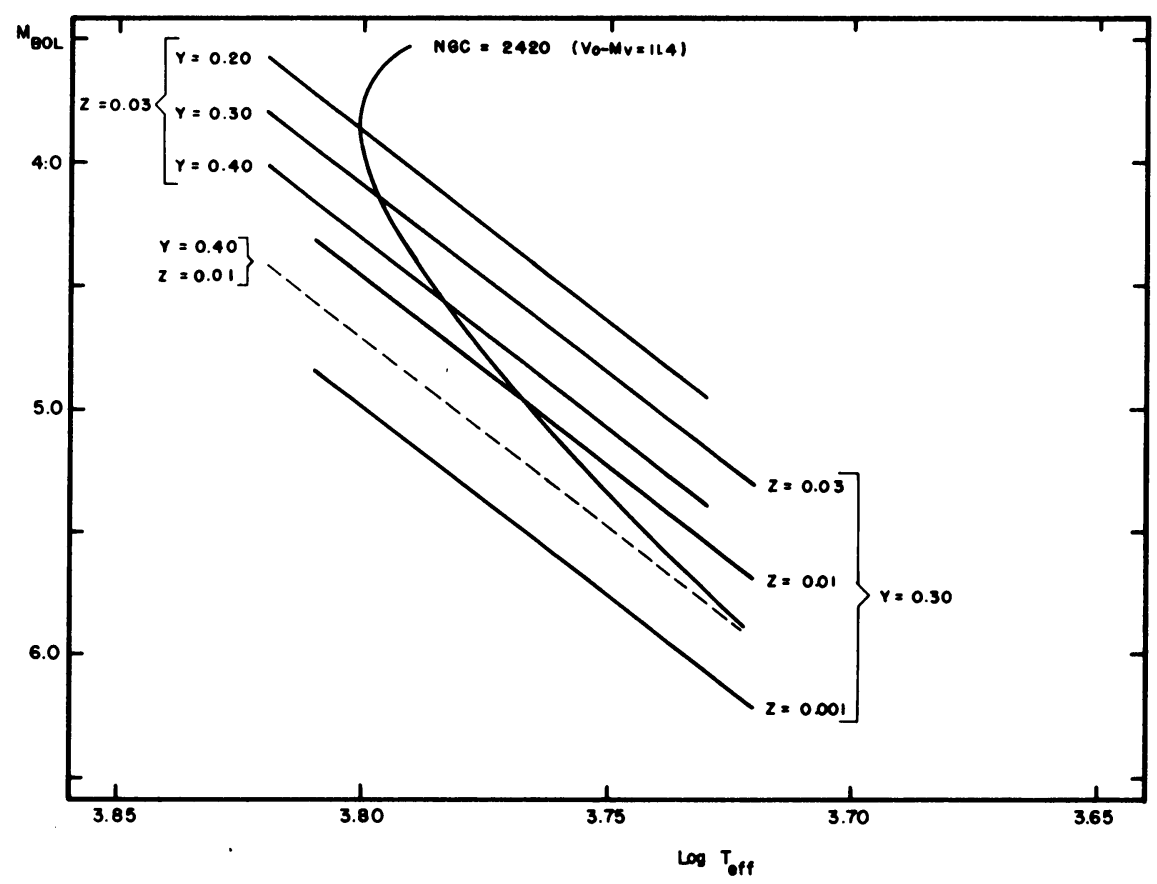

Fig. 6. The position of the zero age main sequence in the $M_{\mathrm{bol}}$-effective temperature plane. There is a substantial dependence on both helium abundance and metal abundance. The three helium abundances are for $Z=0.03$ while the three metal abundances are for $Y=0.30$. Also shown is the observed sequence for NGC 2420 (McClure et al., 1974) and, for comparison, the approximate theoretical position for a sequence with $Y=0.40, Z=0.01$ (dashed line).

\section{Conclusions}

In conclusion, we wish to repeat that the object of this paper has been to describe our work in general terms rather than present any definitive results. We conclude from our exploratory investigations that good $(X, Y, Z)$ values for clusters can be obtained by looking for the interpretation of the observational data that is most consistent with the whole of the results of theoretical models. This result is not surprising since this has been 
exactly the approach used by several authors with respect to the Hyades. What is surprising is how infrequently these methods have been applied to other clusters.

In the present programme two clusters - M 67 and NGC 2420 - have so far been analyzed (Osborn, 1974, 1975). DDO photometry has been obtained for red stars in the fields of 13 additional clusters. The photometry shows that most of these stars are physical cluster members. These data will be discussed in a forthcoming paper.

\section{References}

Gross, P. G.: 1973, Monthly Notices Roy. Astron. Soc. 164, 65.

Janes, K. A.: 1975, Astrophys. J. Suppl. 29, 161.

McClure, R. D.: 1973, in C. Fehrenbach and B. Westerlund (eds.), Spectral Classification and Multicolour Photometry, p. 162.

McClure, R. D., Forrester, W. T., and Gibson, J.: 1974, Astrophys. J. 189, 409.

McClure, R. D. and Racine, R.: 1969, Astron. J. 74, 1000.

McClure, R. D. and van den Bergh, S.: 1968, Astron. J. 73, 313.

Newell, E. B., Rodgers, A. W., and Searle, L.: 1969a, Astrophys. J. 156, 597.

Newell, E. B., Rodgers, A. W., and Searle, L.: 1969b, Astrophys. J. 158, 699.

Osborn, W.: 1973, Astrophys. J. 186, 725.

Osborn, W.: 1974, Monthly Notices Roy. Astron. Soc. 168, 291.

Osborn, W.: 1975, Monthly Notices Roy. Astron. Soc. 172, 631.

Philip, A. G. D.: 1972, Astrophys. J. (Letters) 171, L51.

\section{DISCUSSION}

W. Bidelman: I did not quite understand the origin of the two discrepant values of $Y$ that you quoted.

Osborn: The first value of $Y$ comes from the mean position of the stars in the temperature-gravity diagram (Iigure 4). The second value comes from the position in the temperature $-M_{\text {bol }}$ diagram (Figure 5).

Andersen: I am impressed by the small range you find in $Y$. We have been working on an F-type eclipsing binary where we know the mass to about $2 \%$, the radii to about $1 \%$ from very weli-observed $u v b y$ light curves, the temperature from the accurate mean uvby indices, and the metal abunaance from the $m_{1}$ index, and still we don't trust the resulting value of $Y$ to be better than 0.05 .

Osborn: Of course, I have not put any errors on my values. Let us say that the determination of $Y=0.25$ from Figure 4 and $Y=0.35$ from Figure 5 can be taken to mean that $Y=0.30 \pm 0.05$. There seems to be no parameter that I can change that would give any $Y$ valuc outside this range.

Williams: First, how many colours have you observed and how many parameters do you derive from them?

Secondly, have you observed any branch stars and derived abundances for them?

Osborn: We observe three colours $C(45-48), C(42-45)$, and $C(41-42)$. These are used to derive three physical parameters: effective temperature, surface gravity, and metal abundance. The helium abundance follows from comparison of these three with theoretical models.

No, I have so far observed only clump stars.

Bell: If you change the reddening, do the $Y$ values become in better agreement?

Osborn: In this particular case of NGC 2420 the reddening is quite small, 0.01 or 0.02 depending on who you believe. Therefore, the reddening cannot be decreased significantly. Increasing the reddening increases the discrepancy. 